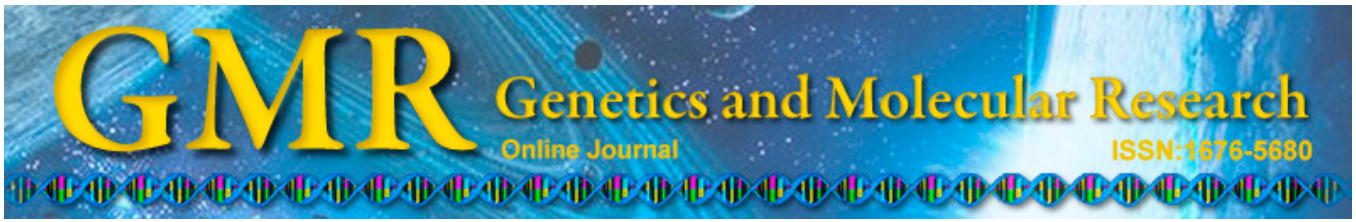

\title{
A cytological study of anther and pollen development in Camellia oleifera
}

\author{
C. Gao, D.Y. Yuan, B.F. Wang, Y. Yang, D.M. Liu and Z.Q. Han
}

Key Laboratory of Cultivation and Protection for Non-Wood Forest Trees of Ministry of Education,

Key Laboratory of Non-Wood Forest Products of Forestry Ministry, Central South University of Forestry and Technology, Changsha, China

Corresponding author: D.Y. Yuan

E-mail: yuandeyi@163.com

Genet. Mol. Res. 14 (3): 8755-8765 (2015)

Received January 23, 2015

Accepted May 8, 2015

Published July 31,2015

DOI http://dx.doi.org/10.4238/2015.July.31.24

\begin{abstract}
The plant species Camellia oleifera is an important producer of edible oil in China. However, it suffers from a low fruit-setting rate. This study used high resolution scanning electron microscopy of semi-thin tissue sections to investigate anther development and pollen formation, and to determine whether problems in forming functional pollen cause low seed setting rates. During anther development, cell sizes within the epidermis and end othecium gradually increase, and at the beginning of the microsporocyte phase, the cells become highly vacuolated. The cell walls of the inner wall cells thicken in a ribbon pattern during the later part of the two-cell pollen stage. Middle layer cells become flattened during the later part of the secondary sporogenous cell stage. A tapetum also forms at this stage; the secondary sporogenous cells begin to degrade at the early microspore stage and completely disappear by the time the pollen is mature, thus forming a glandular tapetum. In microsporocyte, cytokinesis occurs simultaneously with microsporocyte meiosis, and a tetrahedral arrangement is present in tetrads. Reproductive cells begin to form at the later stage of microsporogenesis; the nuclei of
\end{abstract}


the reproductive cells are enlarged as the reproductive cells begin to move away from the pollen wall. Two-cell pollen is first formed after reproductive cells have left the pollen wall, and the number of inclusions in the pollen subsequently increases. The pollen matures for 5-8 days before flowering occurs. No pollen abortion phenomena were observed, suggesting that anther development in C. oleifera is normal and that functional pollen are formed.

Key words: Camellia oleifera; Pollen; Angiosperms; Male gametophyte; Microsporogenesis; Reproduction

\section{INTRODUCTION}

The anther is the male reproductive organ of angiosperms and is the location of male gametophyte and pollen formation. An important tissue of the anther is the anther wall, which is composed, from outside to inside, of the closely located epidermis, endothecium, middle layer, and tapetum. The appearance, structure, and function of these tissue layers are different, and each plays an important role in pollen development (Goldberg et al., 1993). Pollen formation is the most important outcome of the growth and development of anthers; it is the product of a complex and unique process in which somatic cells are transformed into gonial cells that eventually produce haploid microspores after microsporocyte cytokinesis. In the haploid microspore, unequal division results in formation of vegetative cells and reproductive cells that differ in both size and function. Each reproductive cell divides to form two sperm cells in the pollen grain. After maturation, the anther opens and spreads the pollen to complete the development process. During anther development, several specific events occur: first, microsporocytes form a callose wall that degrades subsequently at the tetrad stage; second, large vacuoles are formed in the microspores and these divide into smaller vacuoles by the 2-cell pollen stage; third, the middle layer cells and tapetal cells of the anther wall partially degrade. Different plant species form different anther structures with different developmental characteristics, and these three phenomena exhibit temporal and spatial diversity (Regan and Moffatt, 1990; Kreunen and Osborn, 1999; Wilson and Zhang, 2009; Liu et al., 2011).

Camellia oleifera, commonly known as the tea-oil tree, is an evergreen tree that belongs to the family Theaceae. It is an important woody edible oil plant in South China and is one of the four major woody oil trees of the world, along with oil palm, olive and coconut (Zhuang, 2008). Tea oil extracted from C. oleifera seeds has low cholesterol content, high unsaturated fatty acid content, and other fine features, and forms high-quality edible oil. C. oleifera is mainly distributed in 14 provinces of China in regions south of the Yangtze River. C. oleifera begins to flower in October of each year, and this continues until February of the following year; fruit ripening starts in February and lasts until October. Thus, the reproductive and growth cycle spans both years and seasons. C. oleifera has been grown and used in China for more than 2000 years, but its fruit-setting rate is very low and production yield is very small; these issues have restricted the efficient development of a C. oleifera industry (Gao et al., 2012). There have been few studies on the reproductive and biological characteristics of $C$. oleifera, and consequently, the understanding of the characteristics of zoogamy in this species is limited. Therefore, the effective process of applying theory to agricultural practice for improving production and further study cannot be realized, thus slowing the development of a C. oleifera industry. 
A few studies have reported the embryology of plants belonging to the genus Camellia. In an embryological study of C. oleifera, Cao (1965) described ovule development but did not examine anther development. Tsou (1997) carried out a preliminary study of the embryology of anther and ovule development in three genera of Theaceae (including the genus Camellia) and provided a preliminary description of some embryological features, such as initiation of anther wall formation, the presence of a glandular tapetum, and pollen maturation and diffusion at the flowering phase. Zou et al. (2013) studied basic microsporogenesis and male gametophyte development in Camellia grijsii, and showed that the male gametophyte was fertile and could pollinate at the flowering phase in early February.

Normal anther development and functional pollen formation is a prerequisite and guarantee for $C$. oleifera pollination and fertilization, and is an important aspect to consider when studying whether the cause of the low fruit setting rate in $C$. oleifera is the inability to form normal pollen. Therefore, basic research on C. oleifera anther development is particularly important.

In this study, we investigated anther development in C. oleifera and the associated changes in structure at different times in development. With regard to the relationship between structure and function, we compared anther development and pollen formation processes in $C$. oleifera. The results of this study provide useful information regarding the characteristics of zoogamy in C. oleifera and shed light on the possibility of crossbreeding.

\section{MATERIAL AND METHODS}

\section{Sample collection}

C. oleifera trees were planted in the Central South University of Forestry and Technology campus (latitude $28^{\circ} 8^{\prime} 14^{\prime \prime} \mathrm{N}$, longitude $112^{\circ} 59^{\prime} 32^{\prime \prime} \mathrm{E}$ ) and watered once every fifteen days. The soil was of medium fertility. In 2013 and 2014, adult $C$. oleifera flower buds were collected every five days from July 1 to October 30.

\section{Semi-thin tissue sections}

The anther was carefully removed using tweezers and immediately fixed in $2.5 \%$ glutaraldehyde in $0.1 \mathrm{M}$ phosphate buffer, $\mathrm{pH} 7.2$, for $4 \mathrm{~h}$ at $4{ }^{\circ} \mathrm{C}$. The anther was then washed $3 \mathrm{x}$ $30 \mathrm{~min}$ in $0.1 \mathrm{M}$ phosphate buffer and post-fixed in $1 \%$ osmium tetroxide in $0.1 \mathrm{M}$ phosphate buffer solution for $12 \mathrm{~h}$ at $4^{\circ} \mathrm{C}$. After $3 \times 30$ min washes in $0.1 \mathrm{M}$ phosphate buffer, the tissue was dehydrated through an acetone gradient series, and then embedded in EMbed 812 resin. The tissue block was mounted on an Ultracut R microtome (Leica, Berlin, Germany) and a glass knife was used to cut $1 \mu \mathrm{M}$ sections. The sections were stained with $1 \%$ toluidine blue for $20 \mathrm{~s}$. Sections showing typical developmental stages were selected and analyzed using an optical microscope (BX-51, Olympus, Tokyo, Japan).

\section{Scanning electron microscopy}

The scales of $C$. oleifera flower buds were removed using tweezers and the buds were fixed for $2 \mathrm{~h}$ in $2.5 \%$ glutaraldehyde in $0.1 \mathrm{M}$ phosphate buffer. After washing, the buds were post-fixed in $1 \%$ osmium tetroxide for $2 \mathrm{~h}$. The buds were washed as described above, dehy- 
drated through an ethanol gradient, transitioned into tert-butanol, and lyophilized. The buds were placed into a sample holder and then coated in a sputter coater with gild for $20 \mathrm{~min}$. We used a JSM-6390 scanning electron microscope (JEOL, Tokyo, Japan) to analyze the samples.

\section{RESULTS}

\section{Overview of the anther}

C. oleifera anthers grow on the receptacle to form the androecium, and each anther has four chambers. The anthers have a staggered arrangement and are outside of the pistil (Figure $1 \mathrm{~A}$ and $\mathrm{B}$ ). In cross-section, the anther appears butterfly-shaped, with flat and long connective tissues. A vascular bundle is located centrally, and the left and right sides each contain four symmetrical circular chambers (Figure 1C and D). As C. oleifera has a large anther, we only photographed cross-sections of typical chambers at each developmental stage.
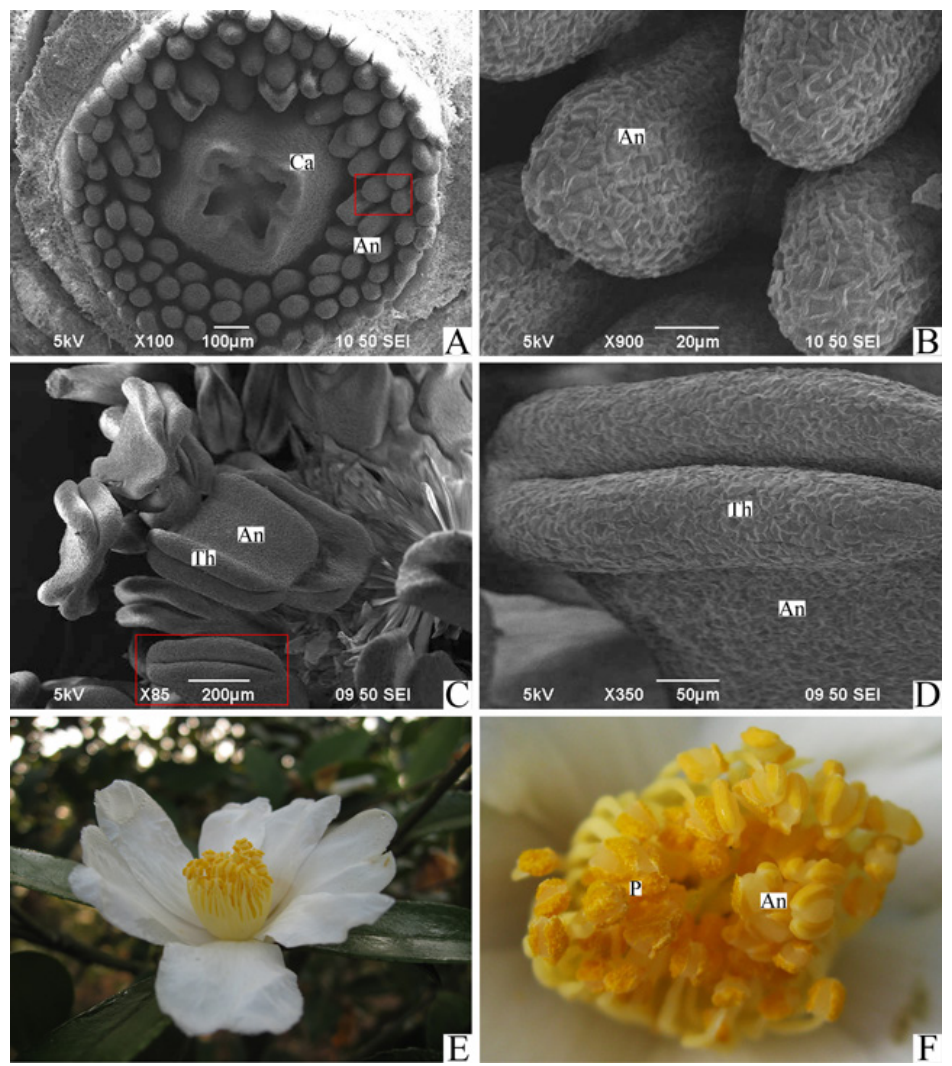

Figure 1. Overview of anther structure in Camellia oleifera. A. An androecium with four chambers. In this example, the four carpels have developed to form a pistil. B. The area outlined by the red box in $\mathbf{A}$ is enlarged and shows a stamen that has not formed a protruding chamber. C. A butterfly-shaped anther has been formed, and the connective tissue is flat and long. D. The area enclosed by the red box in $\mathbf{C}$ is enlarged and shows two protruding chambers at one side of the anther. E. C. oleifera flower. F. Pollen grains are shed from the anthers on the day when the flower blooms. $\mathrm{An}=$ anther; $\mathrm{Ca}=$ carpel; $\mathrm{P}=$ pollen; $\mathrm{Th}=$ theca. 


\section{Sporogenous cell stage}

At the sporogenous cell stage, the archesporial cells of the anther divide and form primary sporogenous cells and primary wall cells (Figure 2A). The primary wall cells continue to go through periclinal and anticlinal divisions to produce an anther wall comprised of 3-4 layers of cells arranged in concentric circles; however, we are currently unable to distinguish between the various components of the anther wall. The primary sporogenous cells continue to undergo mitosis to increase the number of cells (Figure 2B). With the further development of the anther, the concentric arrangement becomes more regular, and all the cell layers in the anther wall can be distinguished. From outside to inside, these layers are epidermis, internal wall of the chamber, middle layer, and tapetum. At this time, cell size and shape differences between the layers are not significant; indeed, the layers are relatively homogeneous and regular, making it difficult to distinguish the number of layers between cell types. The primary sporogenous cells gradually transform into secondary sporogenous cells that have an obvious nucleus, but cell size and shape are still not uniform (Figure 2C). Subsequently, the middle cell shortens radially and lengthens tangentially, to produce a flat shape. The nuclei of tapetal cells increase in size, and the cytoplasm becomes dense and stains darkly with dye. At this point, one can clearly distinguish the components of the C. oleifera anther wall: a layer of epidermis, a layer of internal wall of the chamber, two middle layers, and a layer of tapetum. The secondary sporogenous cells also become larger and regular, and their nuclei are more pronounced. As the anther grows in size, tapetal cells continue to undergo tangential division to increase cell number; the cells lengthen radially and extend toward the inside of the chamber, and their thickness increases. In addition, the secondary sporogenous cells gradually become uniform, and gaps emerge among cells (Figure 2D and E).

\section{Microsporocyte stage}

In anthers at the microsporocyte stage, microsporocyte size increases significantly and large gaps appear among cells inside the chamber. A callose wall appears around the microsporocytes; however, as the gaps between microsporocytes differ, this external callose wall shows an uneven thickness, being thicker at cell corners and thinner elsewhere (Figure 2F). This appearance is characteristic of sporogenous cells and microsporocytes and is used as a classification standard for these cells. At this stage, anther wall cells also show changes. Cells in the outermost layer of the epidermal layer increase in size and become very easy to distinguish. The size of the innermost tapetal cells increases rapidly and almost equals the thickness of the epidermis, endothecium, and the middle layer combined. The nuclei in these tapetal cells are obvious and very easy to distinguish. The cells of the two middle layers vary in size and shape: cells close to the tapetum are narrow, whereas those closer to the endothecium are larger and long. The latter cells and the interior wall cells are both highly vacuolated, and show intense staining of the cell wall and nucleus.

\section{Tetrad stage}

Meiosis in the microsporocytes occurs simultaneously. After meiosis, the resulting four haploid spores have an irregular fan shape and are enclosed in a common callose wall, 
which stains intensely with toluidine blue. The anther wall cells, with the exception of the tapetal cells, have not changed significantly compared to the previous stage. However, the innermost tapetal cells are more closely aligned than before, their size has decreased (i.e., showing a trend to shrink), and they are more irregular in shape. The epidermis of the anther wall, the endothecium, and the middle layer cells become highly vacuolated; the cytoplasm is diminished and is pushed to the periphery of the cells by the large vacuoles (Figure 2G).

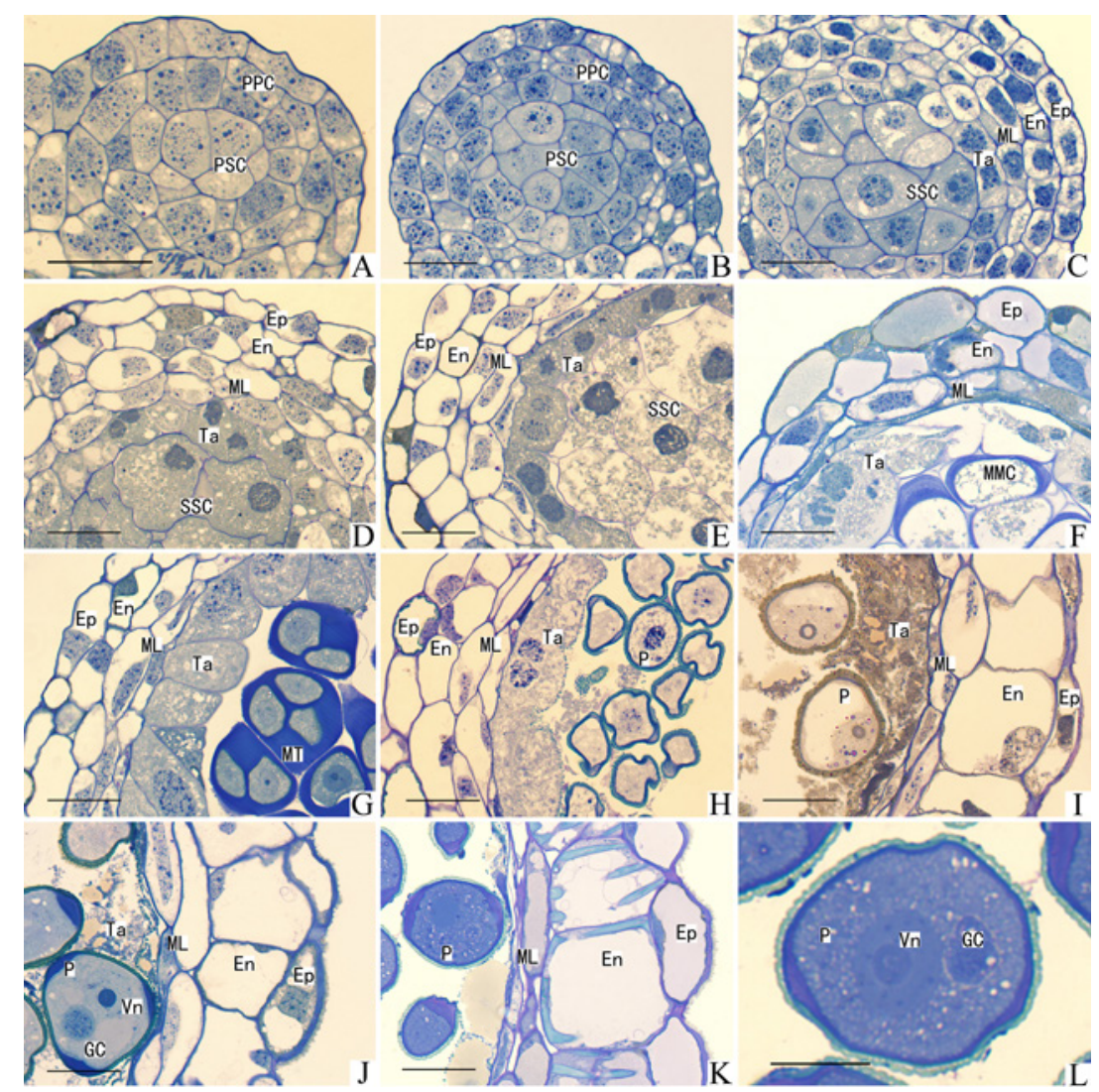

Figure 2. Development of Camellia oleifera anthers. A. Formation of the primary sporogenous cells and primary wall cells. B. Primary sporogenous cells continue to divide. C. The secondary sporogenous cells and all layers of the anther wall form, but the number of layers in the anther wall cannot be determined. D.-E. The anther wall consists of one layer of epidermis, one layer of endothecium, two middle layers, and one layer of tapetum; the secondary sporogenous cells become larger and gaps appear. F. Thicker callose walls appear outside of the pollen mother cells, and the cells in the tapetum have an irregular appearance. G. Tetrad microspores have an irregular appearance. The cytoplasm of the tapetum is dense. H. Microspores are released, and their appearance is irregular with the nucleus located centrally. The cells in the tapetum begin to degenerate. I. The large vacuole inside the microspores pushes the nucleus to the periphery; the tapetal cells disintegrate, and the cells in the endothecium increase in size. J. Microspores divide and form a reproductive cell and a vegetative cell to form the 2-cell pollen; at this stage, the pollen has not been filled. K. When flowering, the radial wall of endothecial cells thickens. L. When the pollen grains are mature, they are filled, and the vegetative cells and sperm cells are visible. En = endothecium; $\mathrm{Ep}=$ epidermis; $\mathrm{GC}=$ generative cell; $\mathrm{ML}=$ middle layer; $\mathrm{MMC}=$ microspore mother cell; $\mathrm{MT}=$ microspore tetrad; $\mathrm{P}=$ pollen; $\mathrm{PPC}=$ primary parietal cell; $\mathrm{PSC}=$ primary sporogenous cell; $\mathrm{SSC}=$ secondary sporogenous cell; $\mathrm{Ta}=$ tapetum; $\mathrm{VN}=$ vegetative nucleus. Bar: $\mathrm{A}-\mathrm{K}=50 \mu \mathrm{M} ; \mathrm{L}=25 \mu \mathrm{M}$. 


\section{Early microspore stage}

With the dissolution of the tetrad callose wall, free microspores are produced. The nucleus of an early microspore is located centrally in the cell. This stage is also known as the mononuclear median period of pollen ( $\mathrm{Hu}, 2005)$. The cytoplasm of free microspores is dense and has an irregular appearance exhibiting various shapes. Initially, there are no obvious vacuoles. Then, as the microspores develop, some small vacuoles appear in the cytoplasm, but the nucleus is still located centrally. In the anther wall tissue, the innermost tapetal cells increase in size, showing a tendency to degrade; the cells become discontinuous with each other, and there is no specific external appearance. Granular materials, most likely the Ubisch bodies synthesized by the pollen external wall, accumulate on the surface of the tangential wall of tapetal cells. Epidermal cells, endothecial cells, and middle layer cells do not change significantly and remain highly vacuolated (Figure $2 \mathrm{H}$ ).

\section{Late microspore stage}

Large vacuoles appear in the advanced stages of development of microspores. The central region of each cell is occupied by this large vacuole, and the nucleus and cytoplasm are pushed to the periphery. This period is called the uninucleate stage of pollen (Hu, 2005). At this stage, microspores begin to resemble pollen grains in shape. The microspores become more regular and develop a spindle shape. The nucleus is usually located on the side of the major axis, indicating that the position of the nucleus of the microspore is controlled. In the anther wall, the cell layers undergo major changes. The most obvious change is that the tapetal cells begin to degenerate, and eventually their cellular structure disappears, their contents degrade, until only cell debris remains. Cells of the middle layer adjacent to the tapetal cells do not change much. The size of the endothecial cells increases in both radial and tangential directions, so that these cells become the largest cells in the anther wall; like the epidermal cells, the endothecial cells remain highly vacuolated (Figure 2I).

\section{Early stage 2-cell pollen}

The microspores undergo mitosis to form a small reproductive cell and a larger vegetative cell, a structure called 2-cell pollen. The reproductive cells are fixed to the pollen inner wall like a lens. As the 2-cell pollen develops, the size of the reproductive cell gradually increases and the nucleus becomes clearer; the reproductive cell moves away from the pollen wall into the cytoplasm of the vegetative cell, thus forming an intracellular cell. With the formation of reproductive cells, the large vacuoles inside the large vegetative cells of the microspores disappear. At the site of the germ pore, the pollen inner wall significantly thickens, and the cells stain more intensely. At this stage, the epidermal cells of the anther wall and the endothecial cells are still highly vacuolated. The cells of the middle layer are retained and do not change significantly; however, the tapetal cells almost completely disappear. At the original location of the tapetal cells, many lipid droplets and very large lipid aggregations are present; these are most likely the products of the degradation of the tapetal cells (Figures $2 \mathrm{~J}$ and $3 \mathrm{~A}-\mathrm{F})$. 


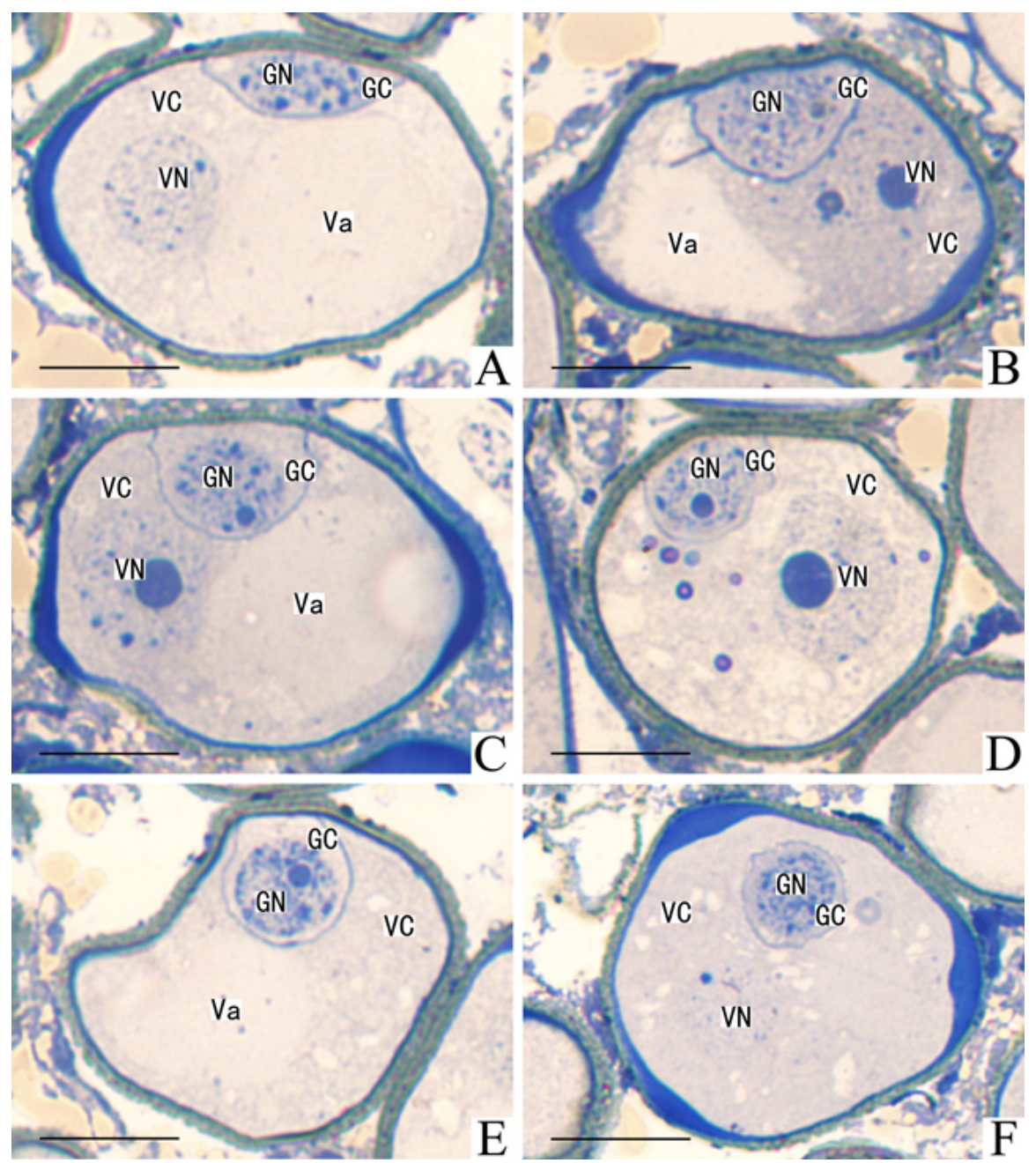

Figure 3. Formation of productive cells inside the Camellia oleifera pollen grain. A.-F. During development, the reproductive cell gradually moves from the pollen inner wall junction toward the interior, and the reproductive nucleus grows larger. Eventually, the entire reproductive cell breaks away from the pollen wall and moves into the cytoplasm of the vegetative cell. The large vacuole in the pollen grain also gradually disappears. GC = generative cell; $\mathrm{GN}=$ generative nucleus; $\mathrm{Va}=$ vacuole; $\mathrm{VC}=$ vegetative cell; $\mathrm{VN}=$ vegetative nucleus. $\mathrm{Bar}=25 \mu \mathrm{M}$.

\section{Pollen maturation stage}

The day before flowering, the pollen is almost mature and is nearly circular in shape. The amount of nutrients accumulating inside the cell increases, the cell stains more intensely over the entire pollen section, and reproductive and vegetative cells are visible (Figure 2L). The mature anther wall is composed of three cell layers: the outermost layer is the epidermis, the middle layer is the endothecium (which has a significantly thickened radial wall), and the innermost layer is the middle layer cells. Some cells in the middle layer show signs of degradation. The tapetal cells have completely disappeared (Figure $2 \mathrm{~K}$ ). 


\section{DISCUSSION}

Sexual reproduction in plants is an integrated process with many interconnected links and failure in any one of these can lead to failure of the whole process. The normal development of anthers and functional pollen formation is a prerequisite for pollination and fertilization in C. oleifera. In this study, we sought to identify whether there was an abortion phenomenon during anther development in C. oleifera and whether this might affect the formation of functional pollen grains and influence the rate of pollination and fertilization. Our analysis of anther development and pollen formation showed that C. oleifera was able to form 2-cell pollen with reproductive functionality, in a similar manner as other species in the genus Camellia that have already been reported (Kapil and Sethi, 1963; Mathew, 1978; Ariyarathna et al., 2011).

In this study, we found that the C. oleifera anther has four chambers and that anther wall development is of the basic type. The anther wall consists of epidermis, endothecium, middle layer, and tapetum, which is consistent with the structures reported in the literature for other species (Goldberg et al., 1993). During development, the development of the anther, and the differentiation of different anther cell tissues, has a great influence on pollen development. Early in the formation of secondary sporogenous cells, cells in the middle layer of the anther wall become elongated in shape. At this stage, we found that the anther wall was composed of five layers of cells: one layer of epidermis, one layer of endothecium, two middle layers, and one layer of tapetum. From the microsporocyte stage to the pollen maturation stage, the epidermis and endothecium cells gradually begin to form vacuoles. From the late microspore stage, the sizes of endothecial cells increases and these reach their maximum after the anther matures. After the formation of 2-cell pollen, the cell walls in the endothecium near the tangential wall begin to thicken, forming a ribbon or fiber pattern that extends outward and upward. Such wall thickening is important to the eventual splitting of the pollen sac $(\mathrm{Hu}, 2005)$. Once microsporocyte cells form, the cells of the middle layer undergo significant changes: they become flat, experience a gradual vacuolization, and then begin to degrade and be absorbed, leaving some remnants in the mature anther. It is worth noting that the innermost layer of the anther, the tapetal cells, are in direct contact with the pollen, and these cells degenerate during a specific period of anther development. Our observations here that the tapetal cells usually have the characteristics of glandular cells, particularly a large nucleus, dense cytoplasm, plentiful organelles, and a polyploid nature, are consistent with those reported by Liu et al. (2011). These characteristics of $C$. oleifera anther development are also similar to those described in C. grijsii (Zou et al., 2013). After secondary sporogenous cells form, tapetal cells form and can be clearly identified. At the microsporocyte stage, the size of tapetal cells increases rapidly and, by the tetrad stage, the thickness of the tapetum is almost equivalent to the total thickness of the other four layers of the anther wall. After the microspores move away from the tetrad, the tapetal cells begin to degenerate and completely disappear by the late microspore stage. C. oleifera tapetal cells have unique temporal and spatial characteristics. The basic features of C. oleifera pollen development are similar to those of C. grijsii (Zou et al., 2013) and Camellia sinensis (Kapil and Sethi, 1963; Tsou, 1997), which also form 2-cell pollen grains. It is noteworthy that the present study gives the first dynamic observation of the two daughter cells that form after the microspore nucleus splits, thereby providing a clear understanding of the process of reproductive cell formation in species belonging to the genus Camellia. After the microspore splits into two daughter nuclei, one moves close to the pollen wall and the other 
moves toward the large vacuole. Subsequently, in quick succession, cytokinesis occurs and an arc-shaped cell plate appears between the two nuclei, splitting the microspore into two cells. The embryological features of $C$. oleifera provide a theoretical basis for the systematic classification of the genus Camellia.

Male sterility is commonly observed in higher plants. The usual manifestation of male sterility is the failure to produce normal anthers, pollen, or male gametes, thus inhibiting complete double fertilization and the ability to form fruit. This sterility is one of the major reasons for low fruit setting rates $(\mathrm{Hu}, 2005)$. Cytological methods offer an important way to study plant anther and pollen development and to determine whether a plant has male sterility. Laser and Lerstern (1972) identified several morphological and anatomical anomalies related to male sterility in higher plants and noted specific unusual behaviors: androecium dysplasia, abnormal microtubule structure, tapetum structure and function abnormalities, endothecium dysplasia, callose deposition and abnormal degradation, microspore development failure, abnormal development after meiosis, and failure of the anther to dehisce. In the present study, we determined that none of these abnormal phenomena occurred during the developmental process of $C$. oleifera anthers and pollen. This species showed formation of functional pollen, indicating normal pollination and fertilization. However, despite the apparent normality of pollen formation, many C. oleifera flowers fail to produce fruit (Gao et al., 2012). As our results preclude the possibility that an inability to form functional pollen is the cause of this low fruit setting rate, other issues that might affect fruit setting in C. oleifera need to be explored, such as macrosporogenesis, female gametophyte developmental conditions, the affinity between the pollen and the pistil, and zygote and embryo development.

\section{Conflicts of interest}

The authors declare no conflict of interest.

\section{ACKNOWLEDGMENTS}

Research supported by the National Natural Science Foundation of China (\#31170639), the Hunan Provincial Innovation Foundation for Postgraduates (\#CX2013A014) and the Graduate Scientific Research Foundation of Central South University of Forestry and Technology (\#CX2013A01). We thank Professor Huiqiao Tian from Xiamen University for assistance with the experiment.

\section{REFERENCES}

Ariyarathna HACK, Gunasekare MTK, Kottawa-Arachchige JD, Paskarathevan R, et al. (2011). Morpho-physiological and phenological attributes of reproductive biology of tea (Camellia sinensis (L.) O. Kuntze) in Sri Lanka. Euphytica 181: 203-215.

Cao HJ (1965). Embryological observation on Camellia oleifera. Acta Bot. Sin. 13: 44-53.

Gao C, Yuan DY, Yuan J, Liao T, et al. (2012). The effect of spraying the nutrient elements and growth regulator at bloom on fruit setting rate of Camellia oleifera. Acta Agric. Univ. Jiangxiensis 34: 0505-0510.

Goldberg RB, Beals TP and Sanders PM (1993). Anther development: basic principles and practical applications. Plant Cell 5: 1217-1229.

Hu SY (2005). Reproductive biology of angiosperms. China Higher Education Press, Beijing, China.

Kapil RN and Sethi SB (1963). Development of male and female gametophytes in Camellia sinensis (L.) O. Kuntze. Proc. Natl. Inst. Sci. India B 29: 574-597. 
Kreunen SS and Osborn JM (1999). Pollen and anther development in Nelumbo (Nelumbonaceae). Am. J. Bot. 86: 16621676.

Laser KD and Lerstern NR (1972). Anatomy and cytology of microsporogenesis in cytoplasmic male sterile angiosperms. Bot. Rev. 38: 425-454.

Liu RS, Qiu YL, Wei DM, Liu HH, et al. (2011). Distribution of starch and neutral lipids in the developing anthers of Ipomoea cairica. Ann. Bot. Fenn. 48: 256-262.

Mathew CJ (1978). Development of male and female gametophytes in Camellia sasanqua. Phytomorphology 28: 262-269.

Regan SM and Moffatt BA (1990). Cytochemical analysis of pollen development in wild-type Arabidopsis and a malesterile mutant. Plant Cell 2: 877-889.

Tsou CH (1997). Embryology of the Theaceae - anther and ovule development of Camellia, Franklinia, and Schima. Am. J. Bot. 84: 369-381.

Wilson ZA and Zhang DB (2009). From Arabidopsis to rice: pathways in pollen development. J. Exp. Bot. 60: 1479-1492. Zhuang RL (2008). Camellia oleifera in China. China Forestry Press, Beijing, 3-4.

Zou F, Yuan DY, Duan JH, Tan XF, et al. (2013). A study of microsporogenesis and male gametogenesis in Camellia grijsii Hance. Adv. J. Food Sci. Technol. 5: 1590-1595. 